\title{
Removal of Aspirated Teeth in a Multiple Trauma Patient, Using Fiberoptic Bronchoscopy With Simultaneous Tracheostomy: Review of 2 Cases
}

\author{
Dong Wook Kim MD DDS, Ji Young Jang MD, Hongjin Shim MD, Ji Ye Jung MD, \\ Eun Young Kim MD, Woong Nam DDS PhD, and Jae Gil Lee MD PhD
}

\begin{abstract}
Aspiration of a tooth in facial trauma is a known complication. There have been few reports on the consequences of maxillofacial trauma, especially when the patient is intubated. We report 2 complicated cases of multiple teeth aspiration and their removal in intubated patients. A special technique using flexible bronchoscopy with simultaneous tracheotomy was used for safe and successful removal of aspirated teeth. We suggest that simultaneous tracheostomy is a safe and effective method for removal of a bronchial foreign body in a maxillofacial traumatized patient. Key words: maxillofacial trauma; tooth aspiration; fiberoptic bronchoscopy. [Respir Care 2014;59(1):e1-e4. (C) 2014 Daedalus Enterprises]
\end{abstract}

\section{Introduction}

Foreign body aspiration is common among children, however, aspiration of a foreign body, especially a tooth, is uncommon among adults. ${ }^{1}$ Despite numerous reports of tooth aspiration and removal, there have been few reports on the consequences of maxillofacial trauma, especially when the patient was intubated. We report 2 successful cases of foreign body (avulsed tooth) removal using fiberoptic bronchoscopy (FOB) with simultaneous tracheostomy in severe maxillofacial trauma patients.

Dr Dong Wook Kim and Dr Nam are affiliated with the Department of Oral and Maxillofacial Surgery, Yonsei University College of Dentistry, Seoul, Korea. Drs Jang and Lee are affiliated with the Division of Surgical Critical Care and Trauma, Department of Surgery, and Dr Jung and Dr Eun Young Kim are affiliated with the Division of Pulmonology, Department of Internal Medicine, Yonsei University College of Medicine, Seoul, Korea. Dr Shim is affiliated with the Department of Surgery, Yonsei University Wonju College of Medicine, Wonju, Korea.

The authors have disclosed no conflicts of interest.

Correspondence: Jae Gil Lee MD PhD, Department of Surgery, Yonsei University College of Medicine, 50 Yonsei-ro, Seodaemun-gu, Seoul, 120-752, Korea. E-mail: jakii@yuhs.ac.

DOI: $10.4187 /$ respcare. 02468

\section{Case Report 1}

A 48-year-old unrestrained male was injured after a motorcycle accident. After arrival at the hospital, endotracheal intubation was attempted to obtain a secure airway, because the patient had severe maxillofacial injury with bleeding and edema. The attempt failed due to poor visibility caused by massive oral bleeding. Continued attempts at cricothyroidotomy and tracheostomy also failed, and, eventually, blind orotracheal intubation with a $7.5 \mathrm{~mm}$ inner diameter endotracheal tube was performed. The patient had an open comminuted fracture of the right mandibular body, a bursting fracture of the ninth thoracic vertebra with a 3-column T-spine fracture, flail chest with multiple fractures of both ribs, pneumomediastinum, and liver contusion. His injury severity score was 22 . Initial chest $\mathrm{x}$-ray before intubation did not show a foreign body in the bronchus. However, a chest $\mathrm{x}$-ray after intubation showed 2 teeth in the right main bronchus (Fig. 1) Chest computed tomography confirmed the location of teeth in the right middle and lower bronchi.

Because the patient had severe maxillofacial injury with bleeding and edema, he was in need of a secure airway, and removal of the teeth with a rigid bronchoscope was impossible. We considered using FOB via the endotracheal tube. In that way, manipulating the foreign body while ventilating seemed possible. However, there was a size discrepancy between the teeth and the endotracheal 


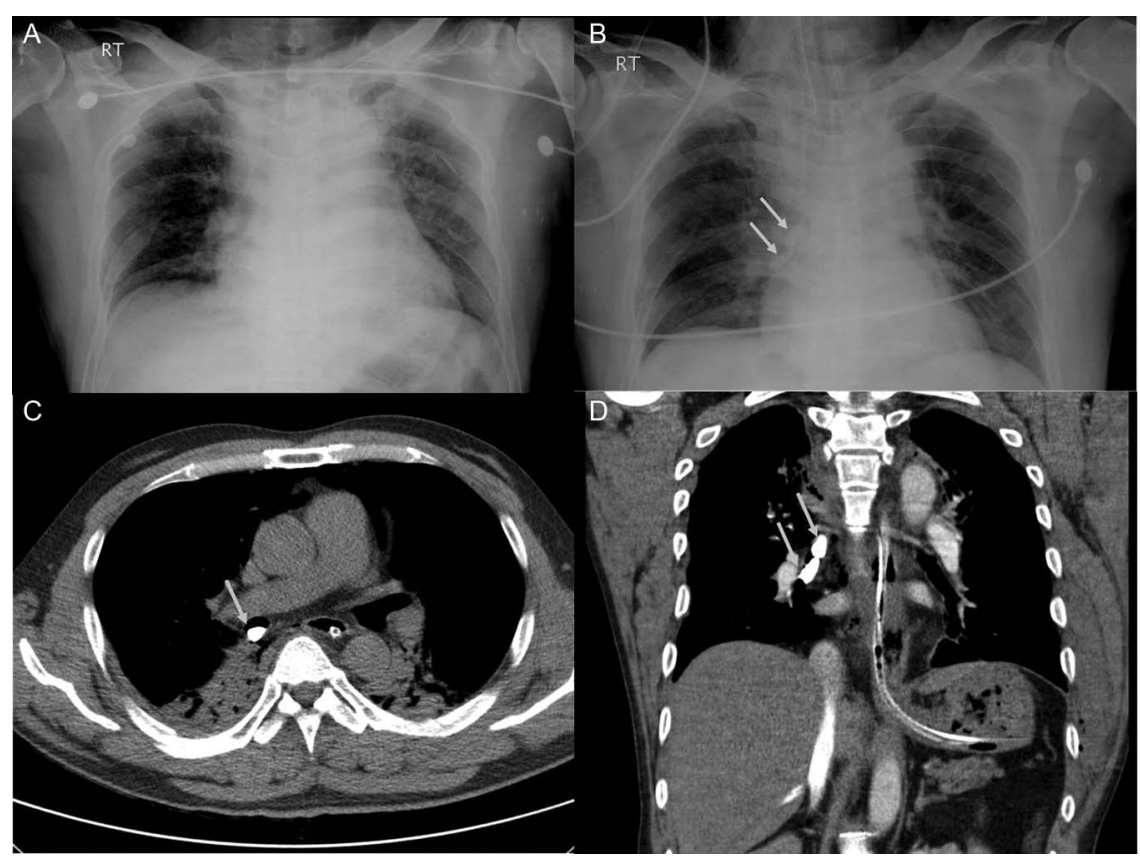

Fig. 1. Patient 1. A: Initial chest x-ray does not show the foreign bodies in the bronchus. After intubation, 2 teeth (arrows) are visible on x-ray in the right bronchus (B). Chest computed tomogram shows the location of teeth in the right bronchus (C and D).

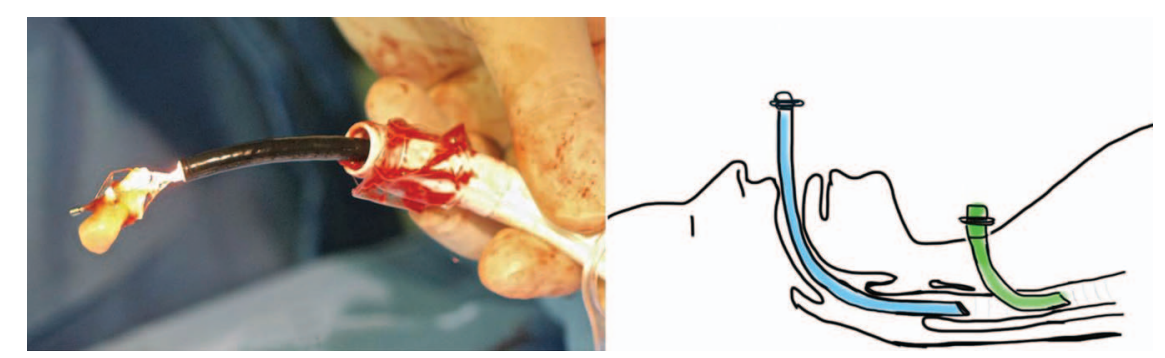

Fig. 2. Left: The tooth was grasped with the basket of a fiberoptic bronchoscope. The tooth was too large to pass through the tracheostomy tube, so the tooth and tracheostomy tube were removed together. Right: Schematic diagram of the procedure. The endotracheal tube is retracted to the level above the tracheostomy. Ventilation and manipulation of the tooth with the bronchoscope is done via the tracheostomy tube. After dragging up the tooth to the tip of tracheostomy tube, mechanical ventilation was switched to the endotracheal tube. The tooth and tracheostomy tube were then removed together.

tube. The 2 teeth measured $25 \times 9 \mathrm{~mm}$ and $27 \times 9 \mathrm{~mm}$, and the inner diameter of the endotracheal tube was $7.5 \mathrm{~mm}$, so extracting the teeth through the tube was impossible without removing the endotracheal tube (Fig. 2A), which would interrupt ventilation and entail the risk of reintubation failure.

We decided to perform tracheostomy as a route for manipulating and removing the teeth, while maintaining the endotracheal tube as a secure route for ventilation. The inner diameter of the tracheostomy tube $(8.0 \mathrm{~mm})$ was slightly larger than the endotracheal tube $(7.5 \mathrm{~mm})$, but still too narrow for the teeth to pass through. That necessitated removal of the tracheostomy tube to remove teeth, but ventilation was possible via the endotracheal tube (see Fig. 2B).
The patient was under volume controlled continuous mandatory ventilation, with PEEP $8.0 \mathrm{~cm} \mathrm{H}_{2} \mathrm{O}$, and $\mathrm{F}_{\mathrm{IO}_{2}} 1.0$, via endotracheal tube, and fully sedated. Tracheostomy was performed as follows. The tracheostomy site was confirmed by trans-illumination of the introduced FOB. The endotracheal tube was retracted above the level of the planned incision site. Open tracheostomy was made using an 8.0 French (inner diameter $8.0 \mathrm{~mm}$ ) tube (Tracoe Pure, Kebomed, Frankfurt, Germany), and mechanical ventilation was done via the tracheostomy. The FOB (BF-XT40, Olympus, Tokyo, Japan) was introduced via the tracheostomy tube. Using an endoscopic basket, one of the teeth was dragged up to the distal end of the tracheostomy tube. The route of mechanical ventilation was then switched to the endotracheal tube during and following removal pro- 


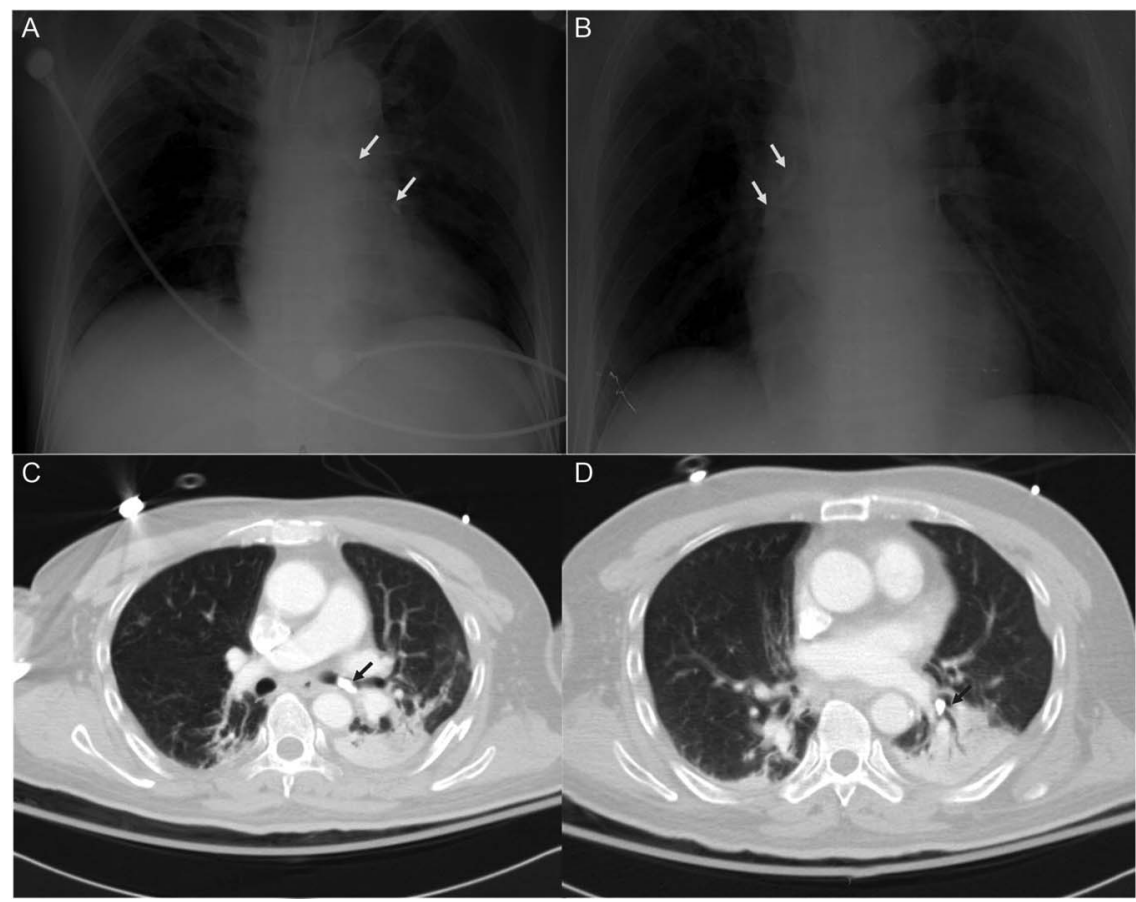

Fig. 3. Patient 2. A: Teeth are located in the left bronchus, and then moved to the right bronchus (B) one day later. C and D: Initial chest computed tomogram shows 2 teeth in the left bronchus.

cedure. The tooth and the tracheostomy tube were pulled out together from the tracheostomy site. The second tooth was removed in the same manner. The total procedure time was 3 hours. Most of the time was spent grasping the teeth, because they were wedged in the bronchus and hard to grasp. After removal of the teeth the tracheostomy tube was re-inserted, and mechanical ventilation resumed through the tracheostomy. The endotracheal tube was then removed. The patient had no substantial air leak or desaturation during the procedure. There was no ventilation interruption during the entire procedure. The chest $\mathrm{x}$-ray showed improvement, and 5 days later, the patient was weaned from mechanical ventilation.

\section{Case Report 2}

A 69-year-old male was severely injured after a fall down the stairs. He was diagnosed with traumatic subarachnoid hemorrhage, diffuse axonal injury, compression fracture of the left temporal bone, lung contusion, multiple rib fractures, avulsion of multiple teeth, and foreign bodies in the bronchus. His injury severity score was 42 . It was also suspected that he had been injured by falling down as a result of a cerebrovascular accident. He was intubated immediately after arrival to the emergency room. The initial chest $\mathrm{x}$-ray showed a tooth-like foreign body in the left bronchus (Fig. 3A) Chest computed tomography showed avulsed teeth in the left bronchus (see Fig. 3C and 3D).

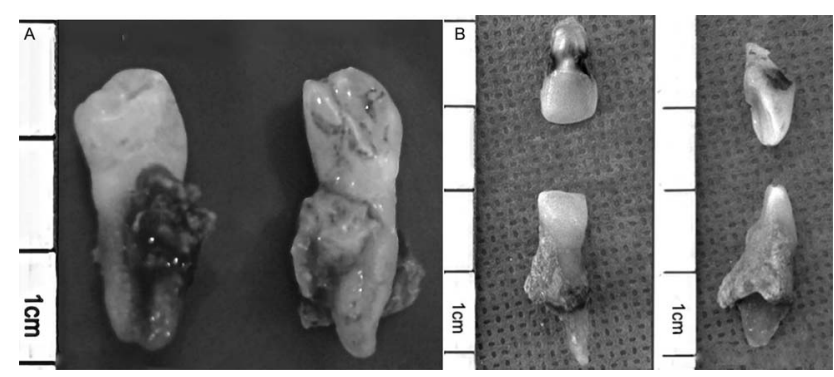

Fig. 4. Removed teeth. A: Patient 1. B: Patient 2.

Migration of the teeth to the right bronchus was noted on follow-up image (see Fig. 3B). He developed pneumonia associated with the tooth, and chest X-ray showed worsening with time. Seven days after admission, the tooth was removed using FOB through the tracheostomy site, as in patient 1. It took 2 hours. Another tooth was observed in the larynx, and removed under the vision of Glide-Scope (Verathon, Bothell, Washington) and a Magill forceps. The patient did well throughout the entire procedure. There was no interruption of ventilation.

The removed teeth are shown in Figure 4.

\section{Discussion}

A rigid bronchoscope is the best option for removal of a foreign body in the trachea and bronchus. A large diameter FOB can be an alternative for a deeply located foreign 
body. In the above-described patients we could not use a rigid bronchoscope, due to the severe maxillofacial injury, which required a definitive airway in patient 1 , and could have worsened the cervical spine injury in patient 2 . Therefore, we had to use an FOB. An FOB with video display can be useful for removal of foreign bodies, especially when the object is stuck and embedded inside the bronchus. $^{2}$

Removing a foreign body with FOB is not simple. Methods include Fogarty catheter, endoscopic forceps, and basket. Using a basket alone may not be sufficient for removal of a foreign body, especially when it is wedged and slippery. Tal-Or et al reported a method with a Fogarty catheter. ${ }^{3}$ They introduced a no. 3 Fogarty catheter through the bronchoscope, passing beyond the tooth, between the gap of the tooth and the luminal wall. Then they inflated the balloon and pulled out the catheter, which pulled the tooth up to the main bronchus. The Fogarty catheter method can be a good option, but it risks trauma to the bronchial wall. ${ }^{4}$

After grasping the foreign body and pulling it up to the tip of the endotracheal tube, one may face the problem that the foreign body is too large to pass through the tube. Some authors have reported failure of removal of a foreign body due to that reason. ${ }^{2}$ Tracheostomy and removing the foreign body through the tracheostomy, by grasping and pulling it out with forceps, might be an option for removing a large foreign body. ${ }^{4}$ There are disadvantages to this method. The tracheostomy tube must be removed during the procedure, and it results in loss of the airway (though it might be temporary). Failure of removal due to poor visibility or rolling and slipping of the foreign body is also possible. In fact, we experienced such a failure in our first attempt in patient 1 . On the first attempt we did prepare for but did not conduct tracheostomy. We first introduced the FOB via the endotracheal tube, dragged the tooth up to the level of the planned tracheostomy site, then tried removing it with Kelly clamp after opening the tracheostomy site wound. That failed due to poor visibility. The tooth was also hard to grasp.

The patient was able to ventilate via the endotracheal tube during the procedure, even when the tracheostomy tube was removed. Tracheostomy enabled a shorter course of visualization and shorter distance of removal. Successful extraction of the tooth was possible by removing it with the tracheostomy tube. We accomplished simultaneous removal of the tooth and tracheostomy tube by continuously pulling the basket, resulting in the tooth being kept against the tip of the tracheostomy tube during the removal.

Whatever the instrument and method used, we suggest that performing a tracheostomy simultaneously while keeping the patient orally intubated is a better option for removal of a foreign body in the bronchus, especially in a patient with maxillofacial trauma. Having 2 routes of approach enables use of an instrument with a larger diameter, which cannot be inserted through the inner channel of the bronchoscope. More importantly, it enables a secure airway and prevents air leak when the patient is mechanically ventilated.

\section{REFERENCES}

1. Baharloo F, Veyckemans F, Francis C, Biettlot MP, Rodenstein DO. Tracheobronchial foreign bodies: presentation and management in children and adults. Chest 1999;115(5):1357-1362.

2. Zhang M, Zhou GJ, Zhao S, Yang JX, Lu X, Gan JX, et al. Delayed diagnosis of tooth aspiration in three multiple trauma patients with mechanical ventilation. Crit Care 2011;15(3):424.

3. Shamim T. Primary intraosseous squamous cell carcinoma of the mandible arising de novo. J Coll Phys Surg Pakistan 2009;19(7): 454-455.

4. Tal-Or E, Schwarz Y, Bloom Y, Kluger Y, Roodik V, Sorkin P. Aspirated tooth removal from airway through tracheotomy and flexible bronchoscopy. J Trauma 1996;40(6):1029-1030. 